\title{
JS-K enhances chemosensitivity of prostate cancer cells to Taxol via reactive oxygen species activation
}

\author{
MINGNING QIU*, SAI ZHANG* , LONGZHI KE, HUANCHENG TANG, XIN ZENG and JIANJUN LIU \\ Laboratory of Urology, Guangdong Medical University, Zhanjiang, Guangdong 524001, P.R. China
}

Received September 20, 2017; Accepted May 15, 2018

DOI: $10.3892 / \mathrm{ol} .2018 .9684$

\begin{abstract}
The aim of the present study was to investigate the influence of the nitric oxide donor prodrug JS-K $\left(\mathrm{C}_{13} \mathrm{H}_{16} \mathrm{~N}_{6} \mathrm{O}_{8}\right)$ on Taxol-induced apoptosis in prostate cancer cells, and to investigate a potential reactive oxygen species (ROS)-associated mechanism. The effect of JS-K on the anticancer activity of Taxol was assessed in prostate cancer cells; cell viability, colony formation, apoptosis, ROS generation and expression levels of apoptosis-associated proteins were investigated. The function of ROS accumulation in the combined effects of JS-K and Taxol was determined using the antioxidant $\mathrm{N}$-acetylcysteine (NAC) and the pro-oxidant oxidized glutathione (GSSG). The results of the present study demonstrated that JS-K was able to increase Taxol-induced suppression of prostate cancer cell proliferation, apoptosis, ROS accumulation and upregulation of apoptosis-associated proteins. Furthermore, NAC reversed the effect of JS-K on Taxol-induced apoptosis and conversely, the pro-oxidant GSSG exacerbated the effect of JS-K on Taxol-induced apoptosis in prostate cancer cells. In conclusion, JS-K enhances the chemosensitivity of prostate cancer cells to Taxol, via the upregulation of intracellular ROS.
\end{abstract}

\section{Introduction}

Taxol, which is a type of microtubule-stabilizing antitumor drug, has a broad antineoplastic spectrum and induces cell apoptosis in various types of human cancer (1). Taxol exerts its anticancer effects via several mechanisms: Inhibition of microtubule assembly and protein isoprenylation, cell cycle arrest at the $\mathrm{G}_{2} / \mathrm{M}$ phase, and stimulation of cell apoptosis and DNA fragmentation $(2,3)$. Taxol has been demonstrated

Correspondence to: Professor Jianjun Liu, Laboratory of Urology, Guangdong Medical University, 57 Renmin Road, Zhanjiang, Guangdong 524001, P.R. China

E-mail: jianjunliulab@163.com

\section{${ }^{*}$ Contributed equally}

Key words: chemosensitivity, Taxol, JS-K, reactive oxygen species, prostate cancer cells to be efficacious for castration-resistant prostate cancer in previous studies $(4,5)$. However, the majority of patients with prostate cancer gradually acquired drug resistance to Taxol following a series of repeated treatments, which may seriously affect their prognosis $(1,6)$. Thus, there is an increasing requirement to develop novel agents which could enhance chemosensitivity to Taxol in patients with prostate cancer.

As an important signaling molecule, antioxidant and toxicant, nitric oxide (NO) is involved in multiple pathological and physiological processes. JS-K $\left(\mathrm{C}_{13} \mathrm{H}_{16} \mathrm{~N}_{6} \mathrm{O}_{8}\right.$; Chemical Abstracts Service no. 205432-12-8), a glutathione transferase-activated nitric oxide-donor prodrug, is reported to promote high intracellular levels of NO and result in cytotoxicity to human prostate cancer cells (7-10). Our previous study demonstrated that JS-K was able to increase the anticancer effects of doxorubicin, which is a type of anthracycline and potent antitumor drug, in human renal carcinoma cells (11). In the present study, the effect of JS-K on the chemosensitivity of human prostate cancer cells to Taxol was investigated. The results of the present study demonstrated that JS-K increased the cytotoxic effects of Taxol on prostate cancer cells. The results of the present study also demonstrated the function of the accumulation of reactive oxygen species (ROS) in apoptosis in prostate cancer cells induced by the combination of JS-K and Taxol. These results revealed the essential functions and mechanisms of JS-K on Taxol-induced apoptosis in prostate cancer cells.

\section{Materials and methods}

Drugs and reagents. Taxol and the nitric oxide prodrug JS-K were purchased from Santa Cruz Biotechnology, Inc. (Dallas, TX, USA), and dissolved in 100\% dimethylsulfoxide (DMSO) as stock solutions (Taxol, $10 \mathrm{mM}$; JS-K, $5 \mathrm{mM}$ ). The final concentration of DMSO did not exceed $0.1 \%$ in any experiment. N-acetylcysteine (NAC) and oxidized glutathione (GSSG) were obtained from Beyotime Institute of Biotechnology (Haimen, China) and dissolved in PBS to concentrations of $100 \mathrm{mM}$ (NAC) and $5 \mathrm{mM}$ (GSSG). Antibodies against BRI1-associated receptor kinase 1 (Bak, cat no. 3814), Bcl-2-associated X protein (Bax, cat no. 2772), B-cell lymphoma (Bcl-2, cat no. 2876), cleaved caspase-3 (cat no. 9661), caspase-7 (cat no. 9494) and caspase-9 (cat no. 9502) were purchased from Cell Signaling Technology, Inc. (Danvers, MA, USA) and an antibody against GAPDH 
was purchased from Abcam (Cambridge, MA, USA) to be used as a loading control. Horseradish peroxidase (HRP) goat anti-rabbit and anti-mouse IgG secondary antibodies were purchased from EarthOx Life Sciences (Millbrae, CA, USA).

Cell lines and cell culture. Human prostate cancer 22RV1 and PC-3 cell lines were purchased from Guangzhou RiboBio Co., Ltd. (Guangzhou, China). 22RV1 cells were cultured in RPMI-1640 medium (Gibco; Thermo Fisher Scientific, Inc., Waltham, MA, USA) and PC-3 cells were cultured in high glucose Dulbecco's modified Eagle's medium (Gibco; Thermo Fisher Scientific, Inc.) at $37^{\circ} \mathrm{C}$ in a humidified atmosphere containing $5 \% \mathrm{CO}_{2}$. All culture media were supplemented with $10 \%$ (v/v) fetal bovine serum (Gibco; Thermo Fisher Scientific, Inc.), $100 \mathrm{U} / \mathrm{ml}$ penicillin and $100 \mathrm{U} / \mathrm{ml}$ streptomycin.

Cell viability assay. The viability of prostate cancer cells was detected using Cell Counting Kit-8 (CCK-8; Dojindo Molecular Technologies, Inc., Kumamoto, Japan) according to the manufacturer's protocol. Briefly, 22RV1 and PC-3 cells were seeded in 96-well plates (NEST Biotechnology Co., Ltd, Jiangsu, China) at a density of $5 \times 10^{3}$ cells/well and then cultured for $24 \mathrm{~h}$. Cells were pretreated with/without JS-K $(1 \mu \mathrm{M})$ for $24 \mathrm{~h}$ and then exposed to Taxol $(2 \mu \mathrm{M})$ for another $24 \mathrm{~h}$, the culture medium was removed and replaced with $100 \mu 1$ medium containing CCK-8 reagent and then incubated at $37^{\circ} \mathrm{C}$ for $2 \mathrm{~h}$. Absorbance was recorded at a wavelength of $450 \mathrm{~nm}$ in a 96-well plate reader (EnSpire 2300 Multilabel Reader; PerkinElmer, Inc., Waltham, MA, USA). Half-maximal inhibitory concentrations $\left(\mathrm{IC}_{50}\right)$ were calculated by probit method using SPSS software (version 18.0; SPSS, Inc., Chicago, IL, USA).

Colony formation assay. Cells were pretreated with/without JS-K $(1 \mu \mathrm{M})$ for $6 \mathrm{~h}$ and then exposed to Taxol $(2 \mu \mathrm{M})$ for another $6 \mathrm{~h}$. Viable cells were harvested and seeded into 6-well plates (NEST Biotechnology Co., Ltd.) at a density of $1.5 \times 10^{3}$ cells/well. Cells were cultured for 2 weeks for assessment of colony formation. Colony formation was analyzed by staining the cells with a Crystal Violet Staining Solution (Beyotime Institute of Biotechnology). Briefly, cells were fixed with $4 \%$ paraformaldehyde for $30 \mathrm{~min}$ at room temperature. Cells were stained with crystal violet staining solution $(10 \mathrm{mg} / \mathrm{ml})$ for $30 \mathrm{~min}$ at room temperature and then washed with ultrapure water. The macroscopic clones $(>1 \mathrm{~mm})$ were photographed with digital camera.

Apoptosis assay. Apoptotic cells were quantified using a fluorescein isothiocyanate (FITC) Annexin V Apoptosis Detection kit (BD Biosciences, Franklin Lakes, NJ, USA) according to the manufacturer's protocol. Briefly, following pretreatment with/without JS-K $(1 \mu \mathrm{M})$ for $24 \mathrm{~h}$ and then exposure to Taxol $(2 \mu \mathrm{M})$ for another $24 \mathrm{~h}, \mathrm{~s}$ the prostate cancer cells were collected, washed with PBS and resuspended in binding buffer. Cells were incubated with annexin V-FITC and propidium iodide for $15 \mathrm{~min}$ at room temperature in the dark, prior to flow cytometric analysis. The stained cells were analyzed by flow cytometry within $1 \mathrm{~h}$.
Measurement of intracellular ROS. To determine the accumulation of intracellular ROS in prostate cancer cells, a ROS assay kit (Beyotime Institute of Biotechnology) was used. Briefly, following pretreatment with/without JS-K $(1 \mu \mathrm{M})$ for $24 \mathrm{~h}$ and then exposure to Taxol $(2 \mu \mathrm{M})$ for an additional $24 \mathrm{~h}$, cells were collected and resuspended with serum-free medium containing dihydrofluorescein diacetate $(10 \mu \mathrm{M})$. Following incubation at $37^{\circ} \mathrm{C}$ for $20 \mathrm{~min}$ in the dark, the cells were harvested and analyzed using flow cytometry with excitation at $488 \mathrm{~nm}$ and emission at $525 \mathrm{~nm}$.

Measurement of mitochondrial membrane potential. 22RV1 and PC-3 cells were seeded into 6-well plates at a density of $3 \times 10^{5}$ cells/well and cultured for $24 \mathrm{~h}$, then were pretreatment with/without JS-K $(1 \mu \mathrm{M})$ for $6 \mathrm{~h}$ and then exposed to Taxol $(2 \mu \mathrm{M})$ for an additional $6 \mathrm{~h}$. The JC-1 Mitochondrial Membrane Potential assay kit (Beyotime Institute of Biotechnology) was used according to the manufacturer's protocol. The cells were analyzed using flow cytometry.

Glutathione assay. Cells were plated in 6-well plates and pretreated with/without JS-K $(1 \mu \mathrm{M})$ for $6 \mathrm{~h}$ and then exposed to Taxol $(2 \mu \mathrm{M})$ for an additional $6 \mathrm{~h}$. The cells were then harvested and lysed by two successive rounds of freezing and thawing. The supernatant was collected by centrifuging at $10,000 \mathrm{x}$ g at $4^{\circ} \mathrm{C}$ for $10 \mathrm{~min}$ and analyzed for reduced glutathione (GSH) and GSSG levels using a GSH and GSSG assay kit (Beyotime Institute of Biotechnology) according to the manufacturer's protocol.

Measurement of adenosine triphosphate (ATP) levels. Intracellular ATP levels were measured using an ATP assay kit (Beyotime Institute of Biotechnology) according to the manufacturer's protocol. Briefly, prostate cancer cells were pretreated with/without JS-K $(1 \mu \mathrm{M})$ for $6 \mathrm{~h}$ and then exposed to Taxol $(2 \mu \mathrm{M})$ for another $6 \mathrm{~h}$ and incubated in $200 \mu \mathrm{l}$ lysis buffer at $4^{\circ} \mathrm{C}$ for $5 \mathrm{~min}$ with gentle shaking. The supernatant of lysis buffer was then collected by centrifugation at $12,000 \mathrm{x} \mathrm{g}$ at $4^{\circ} \mathrm{C}$ for $10 \mathrm{~min}$. Firefly luciferase activity was detected using a Sirius L luminometer (Titertek-Berthold, Pforzheim, Germany) Preferably.

Caspase-Glo 3/7 and Caspase-Glo 9 assays. To examine cell apoptosis following treatment with JS-K and Taxol, caspase-3/7 and caspase-9 activities were analyzed using Caspase-Glo 3/7 and Caspase-Glo 9 assays (Promega Corporation, Madison, WI, USA) according to the manufacturer's protocol. Briefly, cells were seeded into 96-well plates (NEST Biotechnology Co., Ltd.) and pretreated with/without JS-K $(1 \mu \mathrm{M})$ for $24 \mathrm{~h}$ and then exposed to Taxol $(2 \mu \mathrm{M})$ for another $24 \mathrm{~h}$ An equal volume of Caspase-Glo 3/7 or Caspase-Glo 9 reagent was added to each well, and the cells were incubated for $1 \mathrm{~h}$ at room temperature in the dark. The luminescence was measured using a Sirius L luminometer (Titertek-Berthold, Bad Wildbad, Germany).

Reverse transcription-quantitative polymerase chain reaction $(R T-q P C R)$. Total RNA was extracted from 22RV1 and PC-3 cells using an E.Z.N.A. Total RNA Kit I (Omega Bio-tek, Inc. Norcross, GA) and the mRNAs were reversely transcribed into 
stable cDNA with a PrimeScript RT reagent Kit with gDNA Eraser (Takara Bio, Inc., Dalian, China). RT-qPCR analysis was performed by using the SYBR Premix Ex Taq II kit (TaKaRa). The thermocycling conditions included: $95^{\circ} \mathrm{C}$ for $1 \mathrm{~min}$, followed by 40 cycles of amplification at $95^{\circ} \mathrm{C}$ for $5 \mathrm{sec}$ and $60^{\circ} \mathrm{C}$ for $30 \mathrm{sec}$. The levels of mRNAs were expressed as $2^{-\Delta \Delta \mathrm{Cq}}(12)$. The sequences of forward and reverse primers for each gene are listed in Table I.

Western blot analysis. Cells were lysed with radioimmunoprecipitation buffer (Beyotime Institute of Biotechnology) supplemented with $1 \mathrm{mM}$ phenylmethylsulfonyl fluoride (Beyotime Institute of Biotechnology) and proteins were extracted by centrifugation at $10,000 \mathrm{x} \mathrm{g}$ at $4^{\circ} \mathrm{C}$ for $15 \mathrm{~min}$. Total protein was determined by a BCA Protein assay kit (Beyotime Institute of Biotechnology). Proteins (40 $\mu \mathrm{g} / \mathrm{lane})$ were loaded onto SDS-PAGE gels (12\%) then transferred onto polyvinylidene difluoride membranes (Merck KGaA, Darmstadt, Germany), which were blocked with 5\% non-fat milk in Tris-buffered saline and 1\% Tween 20 (TBS-T) and incubated with the aforementioned primary antibodies $(1: 1,000)$ in diluent (5\% BSA in TBS-T) overnight at $4^{\circ} \mathrm{C}$. Following six washes with TBS-T for 5 min each, the membranes were probed with HRP-conjugated goat anti-rabbit or anti-mouse IgG secondary antibody (\#E030120, \#E030110; EarthOx Life Sciences, Millbrae, CA, USA; dilution 1:10,000 in TBS-T containing $5 \% \mathrm{BSA}$ ) for $2 \mathrm{~h}$ at room temperature.

Statistical analysis. All experiments were performed in triplicate. The results are presented as the mean \pm standard deviation. All data were analyzed using one-way analysis of variance using SPSS software (version 18.0; SPSS, Inc., Chicago, IL, USA). Differences between treatments were assessed using a Fisher's least significant difference test. $\mathrm{P}<0.05$ was considered to indicate a statistically significant difference.

\section{Results}

$J S$-K promotes Taxol-induced suppression of proliferation and apoptosis of prostate cells. Prostate cancer cells were exposed to Taxol, JS-K or their combination for $24 \mathrm{~h}$. A CCK-8 assay (Fig. 1A) and a colony formation assay (Fig. 1B and C) were performed to evaluate the effects of Taxol and JS-K on prostate cancer cells, and the data indicated that the number of viable cells was significantly decreased by treatment with Taxol and JS-K together compared with Taxol alone. The half-maximal inhibitory concentrations of Taxol were significantly decreased when cells were pretreated with JS-K $(1 \mu \mathrm{M})$ for $24 \mathrm{~h}$ (Fig. 1D). These data suggested that JS-K was able to significantly promote Taxol-induced suppression of prostate cancer cell proliferation. The apoptosis-inducing effect of Taxol, JS-K and their combination was investigated. It was revealed that JS-K significantly increased apoptosis (Fig. 1E) in Taxol-treated prostate cancer cells. These results indicated that JS-K significantly increased Taxol-induced suppression of proliferation and apoptosis of prostate cancer cells.

JS-K increases Taxol-induced ROS production and decreases the Taxol-induced decrease in the GSH/GSSG ratio in
Table I. Sequences for target gene primers.

\begin{tabular}{lll}
\hline Gene & \multicolumn{1}{c}{ Primer sequence (5'-3') } & $\mathrm{T}_{\mathrm{m}},{ }^{\circ} \mathrm{C}$ \\
\hline \multirow{2}{*}{ GAPDH } & TGCACCACCAACTGCTTAG (F) & 60.07 \\
& AGTAGAGGCAGGGATGATGTTC (R) & 59.72 \\
\multirow{2}{*}{ Bak } & CCCAACCCATTCACTACAGG (F) & 59.80 \\
& CCCACTTAGAACCCTCCAGA (R) & 59.80 \\
\multirow{2}{*}{ Bcl-2 } & CTTTGAGTTCGGTGGGGTCA (F) & 59.80 \\
& GGGCCGTACAGTTCCACAAA (R) & 59.80 \\
\multirow{2}{*}{ Bax } & AAGCTGAGCGAGTGTCTCAAG (F) & 60.00 \\
& CAAAGTAGAAAAGGGCGACAAC (R) & 58.20
\end{tabular}

$\mathrm{T}_{\mathrm{m}}$, melting temperature; Bak, BRI1-associated receptor kinase 1; Bcl-2, B-cell lymphoma 2; Bax, Bcl-2-associated X protein; $\mathrm{F}$, forward; $\mathrm{R}$, reverse.

prostate cancer cells. The effects of JS-K on Taxol-induced ROS production were assessed; it was revealed that ROS production was significantly increased in prostate cancer cells that were treated with Taxol and JS-K together (Fig. 2A). The levels of GSH and GSSG were investigated; it was revealed that the GSH/GSSG ratio was significantly decreased in cells treated with Taxol and JS-K together compared with Taxol alone (Fig. 2B).

JS-K exacerbates the Taxol-induced decrease in mitochondrial membrane potential and ATP levels in prostate cancer cells. The Taxol and JS-K-induced increase in total ROS and decrease in GSH/GSSG ratio (Fig. 2A and B) may contribute to increased mitochondrial dysfunction and lead to mitochondria-mediated apoptosis. To investigate the dysfunction in mitochondrion, the mitochondrial membrane potential and intracellular levels of ATP in prostate cancer cells treated with JS-K and Taxol was determined. It was revealed that the mitochondrial membrane potential (Fig. 2C) and intracellular levels of ATP (Fig. 2D) was significantly decreased in cells treated with Taxol and JS-K together compared with Taxol alone.

$J S-K$ and Taxol synergistically regulates the apoptosisassociated signaling pathway. In the present study, prostate cancer cells were treated with Taxol, JS-K or their combination for $24 \mathrm{~h}$. mRNA (Fig. 3A and B) and protein (Fig. 3C and Table II) levels of Bax and Bak were upregulated in response to the combination of Taxol and JS-K, whereas Bcl-2 levels were downregulated. The levels and activities of caspase-3/7/9 were investigated; increased levels of cleaved caspase-3, cleaved caspase-7 and cleaved caspase-9 (Fig. 3C) and increased activity of caspase-3/7 (Fig. 3D) and caspase-9 (Fig. 3E) in cells treated with Taxol and JS-K together compared with the cells treated with Taxol alone was revealed.

$N A C$ reverses $J S$ - $K$-induced anticancer activity of Taxol and $G S S G$ exacerbates the effects of $J S-K$. To investigate the effects of ROS on Taxol and JS-K-induced prostate cancer cell proliferation suppression and apoptosis, prostate cancer cells were treated with Taxol and JS-K in the presence or 


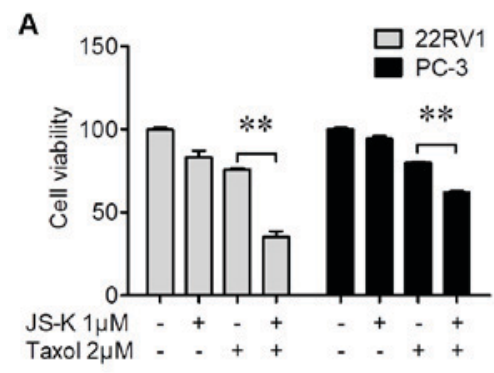

C

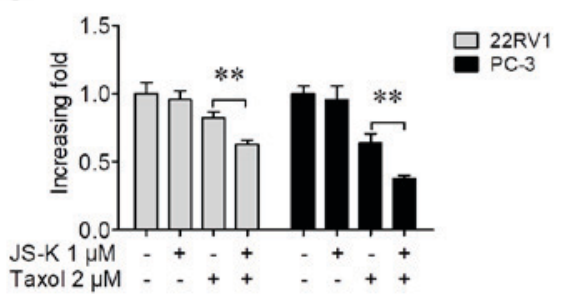

B

22RV1
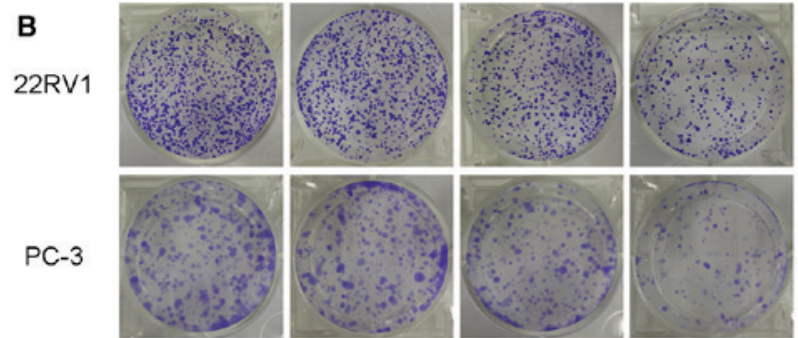

JS-K $1 \mu \mathrm{M}$

Taxol $2 \mu \mathrm{M}$

$+$

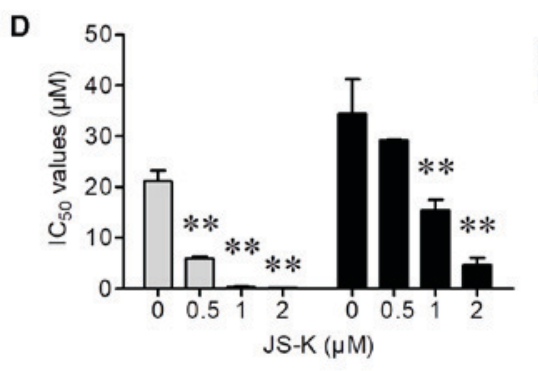

$\square$ 22RV1

- $\mathrm{PC}-3$

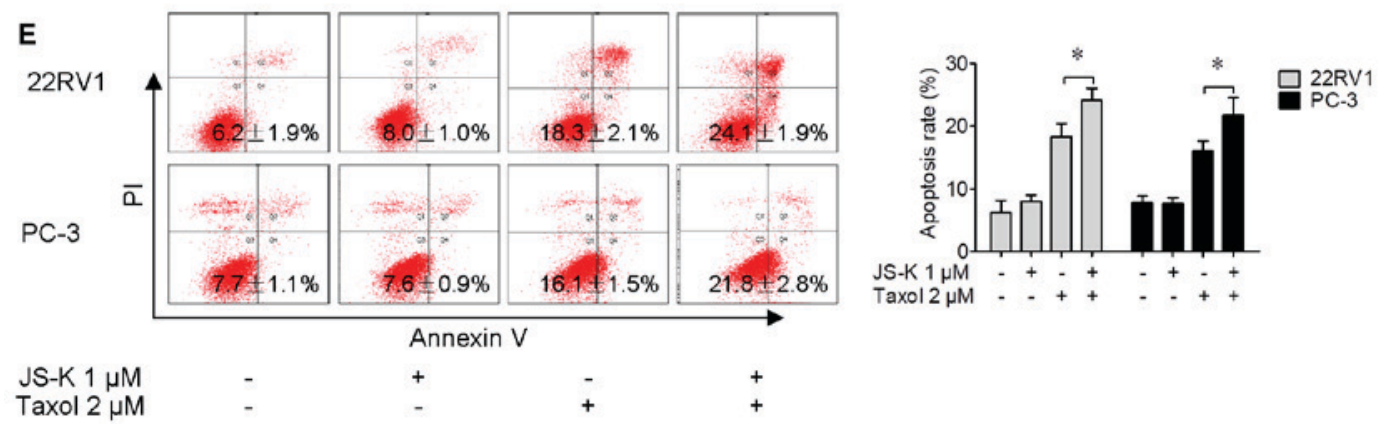

Figure 1. JS-K promotes Taxol-induced suppression of prostate cancer cell proliferation and apoptosis. (A) Cell viability following treatment with JS-K and Taxol was determined using a Cell Counting Kit-8 assay. (B) Representative images and (C) cumulative data from colony formation assay following

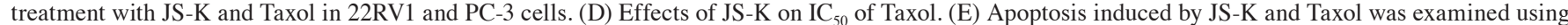
FITC-annexin V/PI staining; representative dot plots (left panel) and cumulative data (right panel). The results are presented as the mean \pm SD for three independent experiments. ${ }^{*} \mathrm{P}<0.05,{ }^{* *} \mathrm{P}<0.01$. $\mathrm{IC}_{50}$, half-maximal inhibitory concentration; FITC, fluorescein isothiocyanate; PI, propidium iodide.
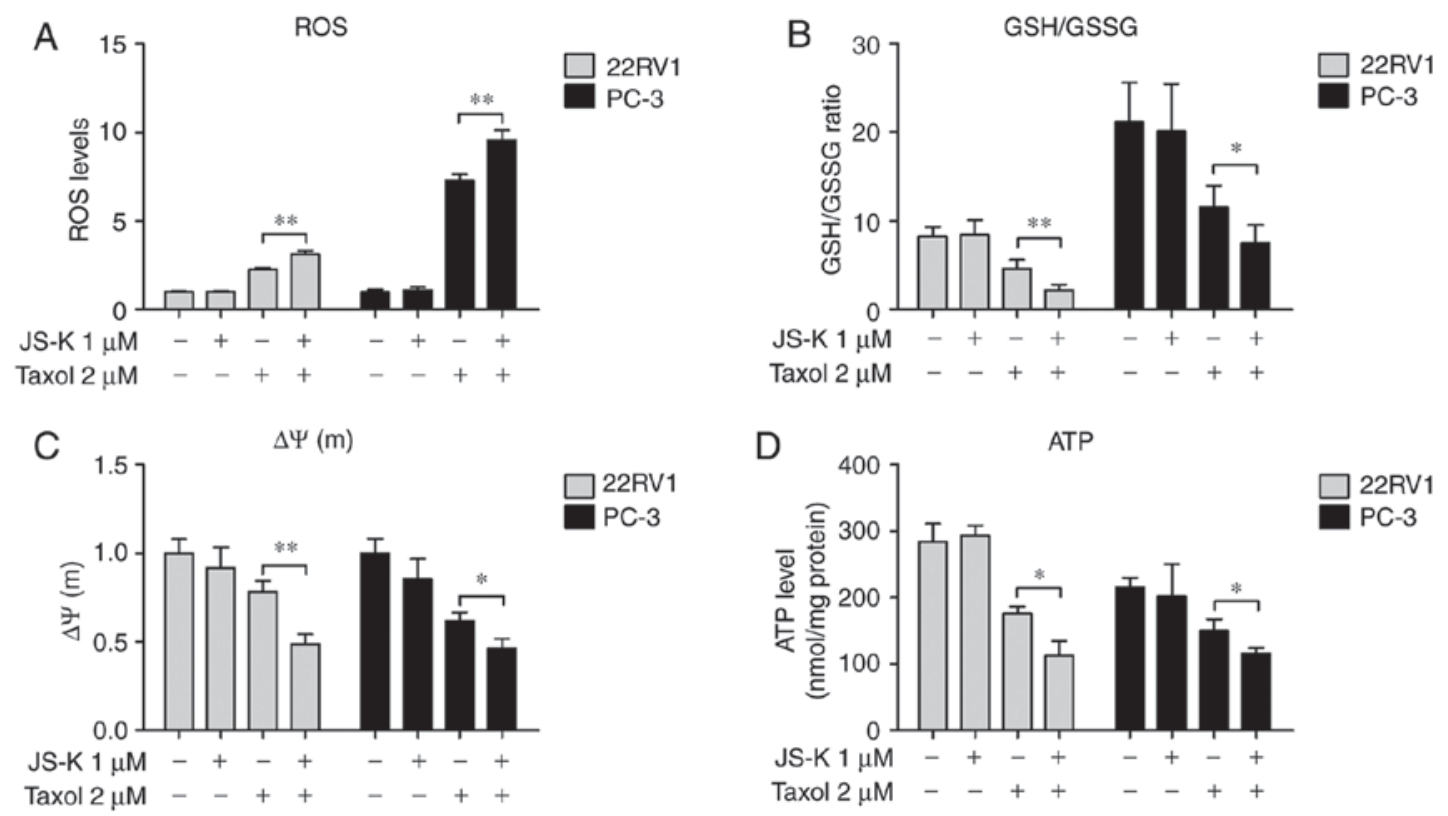

Figure 2. Synergistic effects of JS-K and Taxol on ROS production, GSH/GSSG ratio, mitochondrial membrane potential and ATP levels in prostate cancer cells. Prostate cancer cells were treated with JS-K and Taxol for $6 \mathrm{~h}$ and (A) ROS generation, (B) GSH/GSSG ratio, (C) mitochondrial membrane potential and (D) ATP levels were determined. The data are presented as the mean \pm standard deviation for three independent experiments. ${ }^{*} \mathrm{P}<0.05$, ${ }^{* *} \mathrm{P}<0.01$. GSH, reduced glutathione; GSSG, oxidized glutathione; ATP, adenosine triphosphate; ROS, reactive oxygen species; $\Delta \Psi(\mathrm{m})$, mitochondrial membrane potential. 
Table II. Expression levels of related proteins following treatment with JS-K and Taxol.

Expression levels of 22RV1

\begin{tabular}{|c|c|c|c|c|c|c|c|c|}
\hline \multirow[b]{2}{*}{ Treatment } & & \\
\hline & Control & JS-K & Taxol & JS-K+Taxol & Control & JS-K & Taxol & JS-K+Taxol \\
\hline Bak & 1.00 & 1.06 & 1.68 & 3.74 & 1.00 & 1.23 & 2.04 & 3.12 \\
\hline Bax & 1.00 & 1.01 & 1.26 & 1.82 & 1.00 & 1.11 & 4.56 & 6.44 \\
\hline $\mathrm{Bcl}-2$ & 1.00 & 0.85 & 0.62 & 0.62 & 1.00 & 0.81 & 0.65 & 0.79 \\
\hline Cl-caspase-3 & 1.00 & 1.10 & 1.72 & 2.46 & 1.00 & 1.37 & 2.21 & 3.42 \\
\hline Caspase-7 (35 kDa) & 1.00 & 1.68 & 157 & 1.81 & 1.00 & 0.92 & 1.02 & 1.21 \\
\hline Caspase-7 (30 kDa) & - & - & - & - & 1.00 & 1.32 & 2.26 & 2.06 \\
\hline Caspase-7 (20 kDa) & 1.00 & 2.56 & 2.76 & 2.76 & 1.00 & 4.35 & 13.04 & 21.74 \\
\hline Caspase-9 (47 kDa) & 1.00 & 0.89 & 0.70 & 0.84 & 1.00 & 1.40 & 1.57 & 1.83 \\
\hline Caspase-9 (37 kDa) & 1.00 & 0.89 & 1.00 & 1.53 & 1.00 & 2.04 & 2.54 & 2.71 \\
\hline
\end{tabular}

A

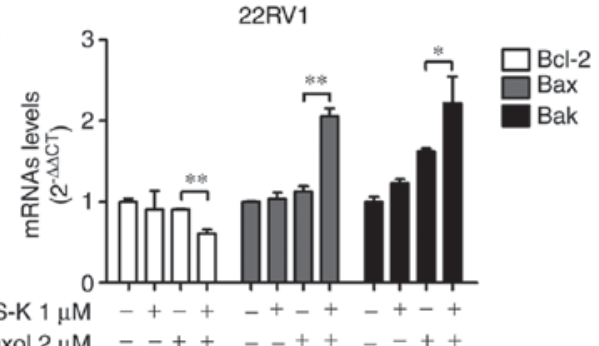

Taxol $2 \mu \mathrm{M}--++--++--+$
B

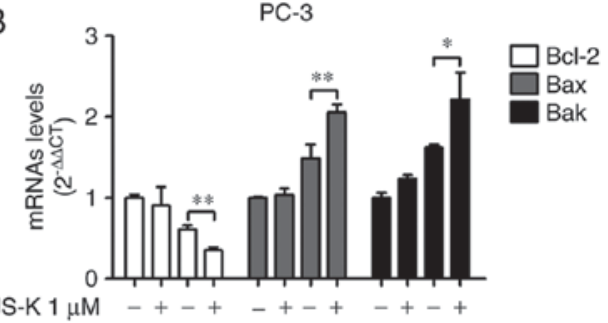

Taxol $2 \mu \mathrm{M}--++--++-++$

C

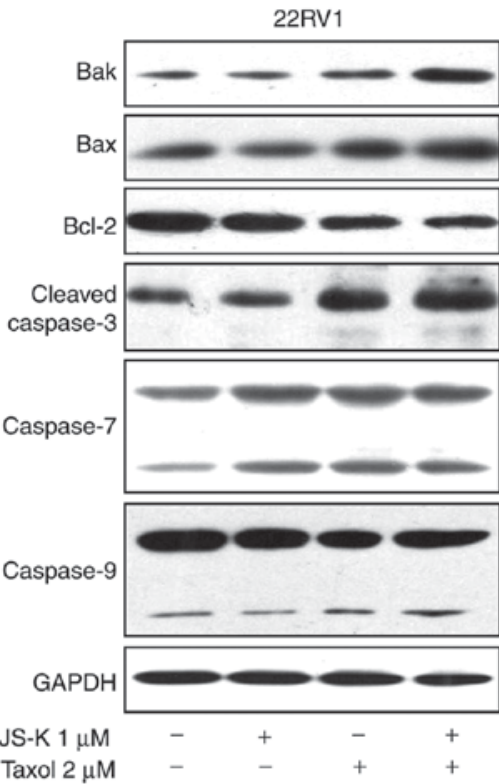

$\mathrm{D}$

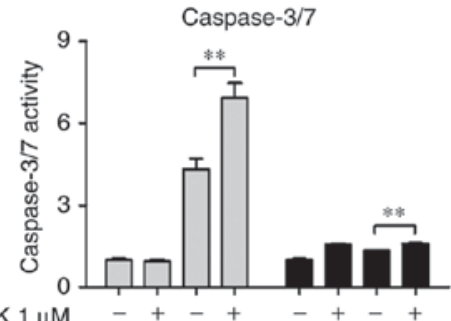

JS-K $1 \mu \mathrm{M}$

Taxol $2 \mu \mathrm{M}$
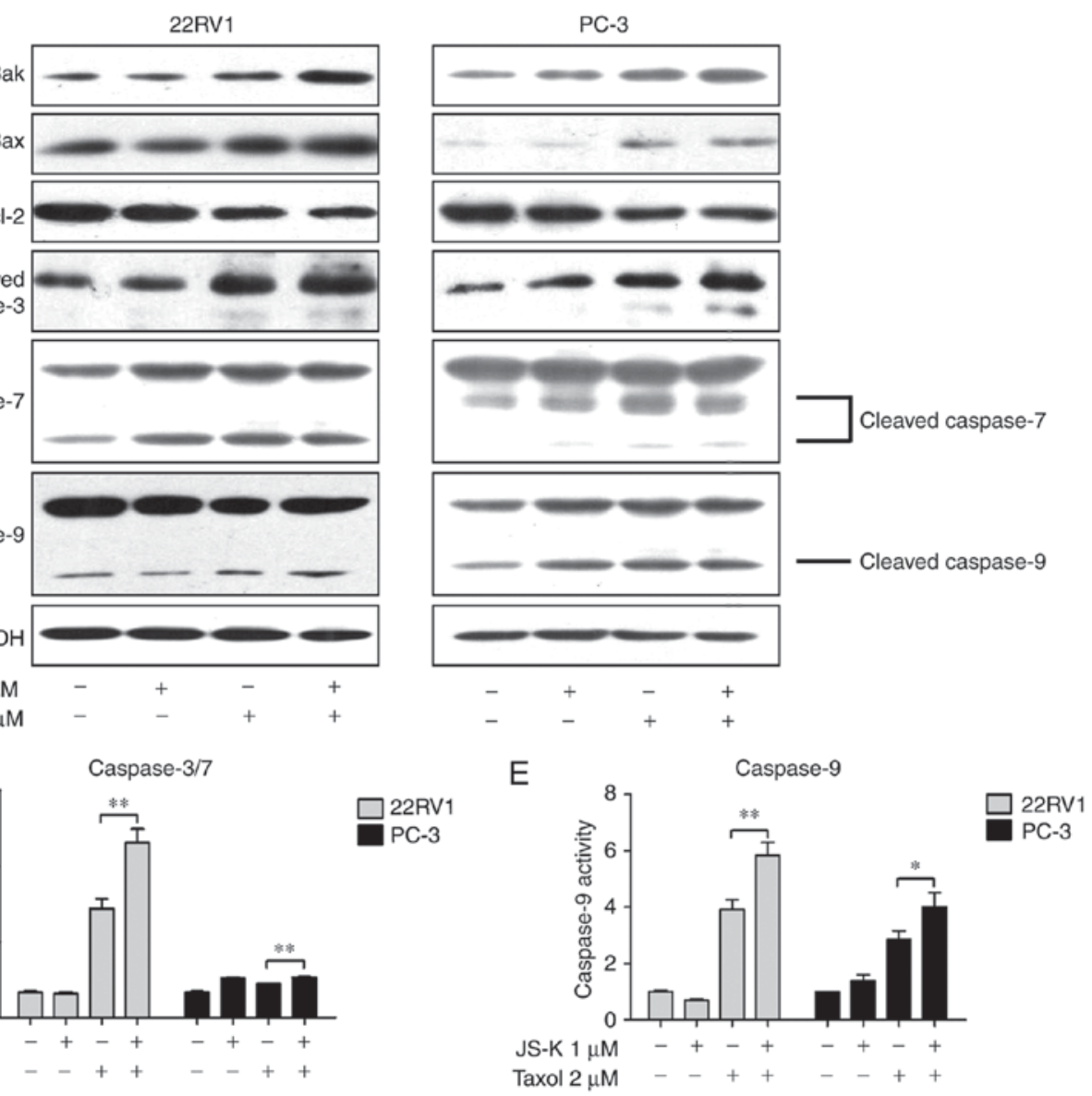

Figure 3. Effects of JS-K and Taxol on the apoptosis-associated signaling pathway. mRNA levels of Bcl-2, Bax and Bak in (A) 22RV1 and (B) PC-3. (C) Expression levels of apoptosis-associated proteins. (D) Activity of caspase- $3 / 7$ and (E) activity of caspase- 9 . The data are presented as the mean \pm standard deviation for three independent experiments. ${ }^{*} \mathrm{P}<0.05,{ }^{* * *} \mathrm{P}<0.01$. Bak, BRI1-associated receptor kinase 1; Bax, Bcl-2-associated $\mathrm{X}$ protein; Bcl2, B-cell lymphoma 2; ROS, reactive oxygen species; ATP, adenosine triphosphate; GSH, reduced glutathione; GSSG, oxidized glutathione. 
A

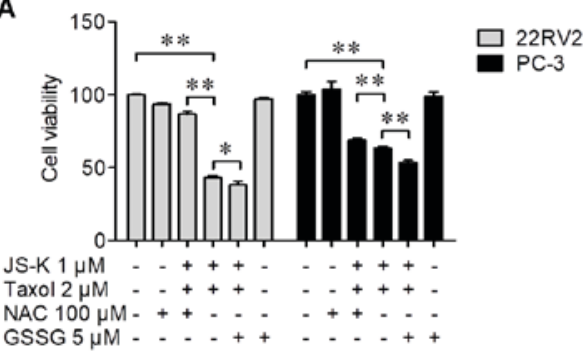

C

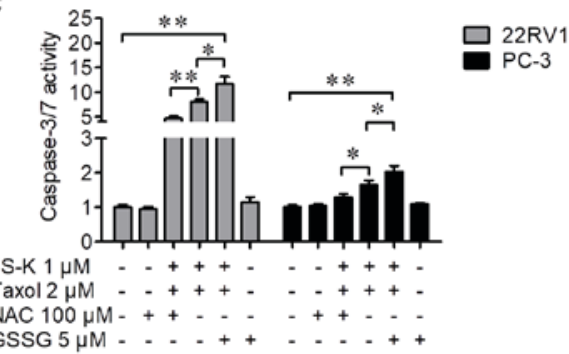

B

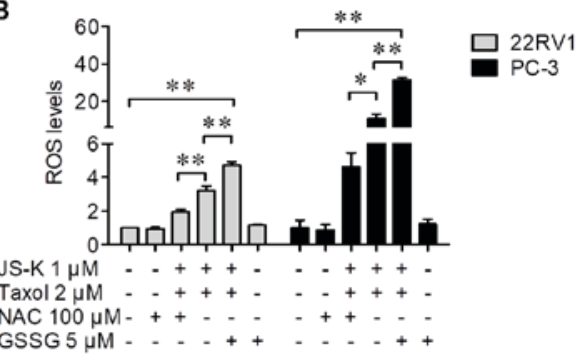

D

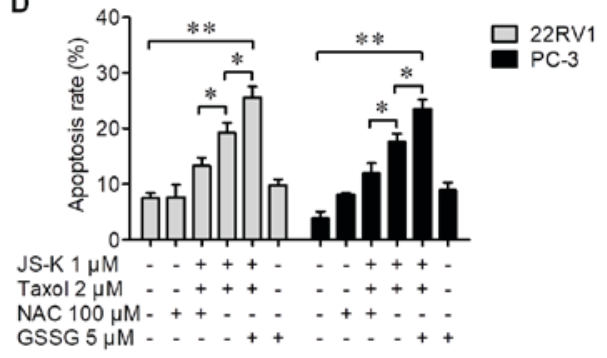

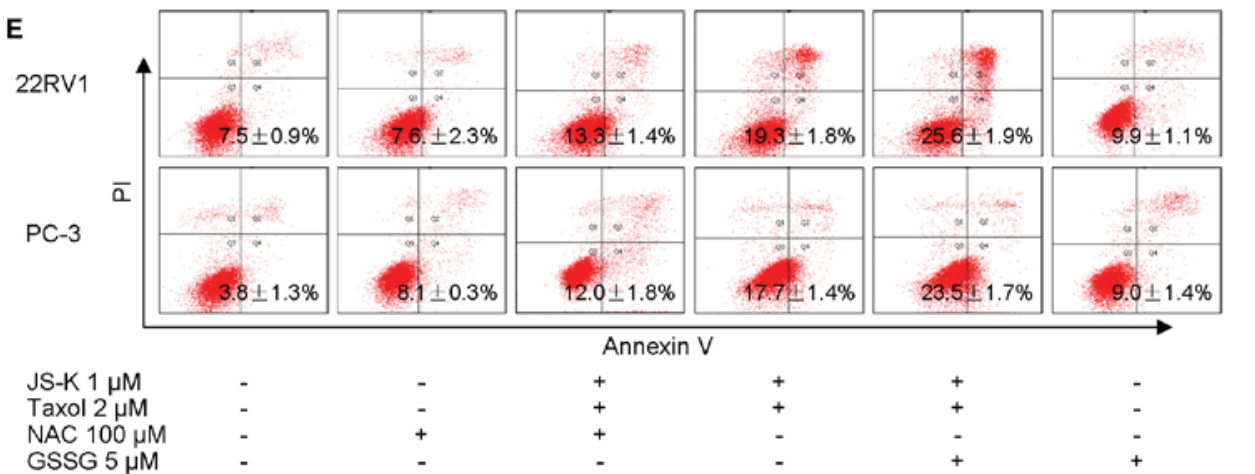

Figure 4. Effects of NAC and GSSG on JS-K- and Taxol-induced apoptosis in prostate cancer cells. (A) cell viability was measured using a Cell Counting Kit-8 assay. (B) Accumulation of intracellular ROS in prostate cancer cells. (C) Caspase-3/7 activity of 22RV1 and PC-3 cells. (D) Apoptosis of prostate cancer cells treated by JS-K and Taxol with or without NAC $(100 \mu \mathrm{M})$ and GSSH $(5 \mu \mathrm{M})$ were analyzed using FITC-annexin V/PI staining. Cumulative results are presented. (E) Representative dot plots of apoptosis of prostate cancer cells. The data are presented as the mean \pm standard deviation for three independent experiments. "P<0.05, ${ }^{* *} \mathrm{P}<0.01$. NAC, $\mathrm{N}$-acetylcysteine; GSSG, oxidized glutathione; ROS, reactive oxygen species; FITC, fluorescein isothiocyanate; PI, propidium iodide; $\mathrm{A}$, annexin $\mathrm{V}$.

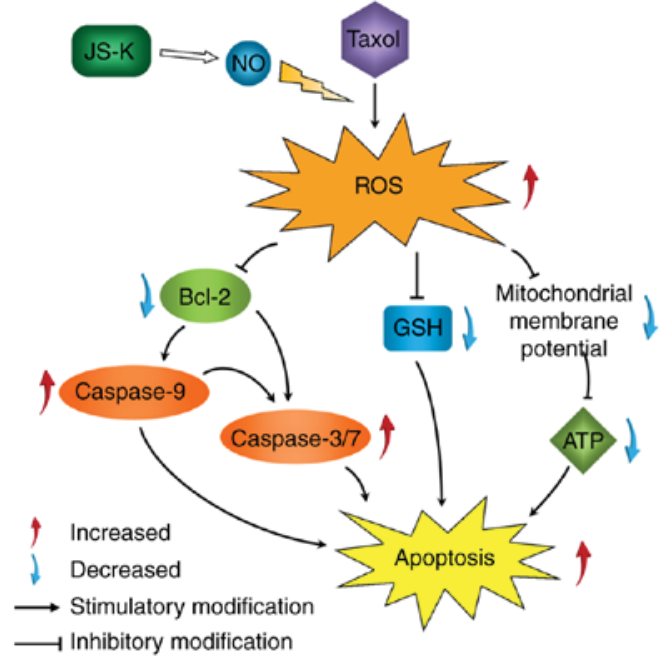

Figure 5. Proposed cellular pathway of JS-K on Taxol-induced cell apoptosis in prostate cancer cells. JS-K promoted Taxol-induced prostate cancer cell apoptosis via the activation of caspase-3/7 and caspase-9 and suppression of ATP and GSH/GSSG ratio. The production of ROS induced by Taxol increased, which eventually led to apoptosis. ATP, adenosine triphosphate; GSH, reduced glutathione; GSSG, oxidized glutathione; ROS, reactive oxygen species; Bcl-2, B-cell lymphoma 2; Bax, Bcl-2-associated X protein. absence of the antioxidant NAC $(100 \mu \mathrm{M})$ or pro-oxidant GSSG $(5 \mu \mathrm{M})$ for $24 \mathrm{~h}$. Cells treated with JS-K and Taxol together was demonstrated to decrease the number of viable cells (Fig. 4A) and increase ROS levels (Fig. 4B), caspase 3/7 activity (Fig. 4C) and apoptosis (Fig. 4D and E) in prostate cancer cells; whereas NAC $(100 \mu \mathrm{M})$ reversed the JS-K-induced increase in chemosensitivity of cells to Taxol (Fig. 4A-D). Furthermore, GSSG $(5 \mu \mathrm{M})$ exacerbated the JS-K-induced increase in chemosensitivity of cells to Taxol (Fig. 4A-D). These results indicated the functions of ROS in JS-K-sensitized Taxol exerting anticancer activity in human prostate cancer cells.

\section{Discussion}

Taxol is recognized as an effective anticancer drug for treating castration-resistant prostate cancer and has been clinically challenged by its acquisition of drug resistance following repeated treatments $(1,4,6)$. In the present study, it was indicated that JS-K increased Taxol-induced proliferation suppression and apoptosis in prostate cancer cells. Taxol was identified to increase ROS generation in cancer cells (13). Our 
previous study demonstrated that JS-K promotes apoptosis in prostate cancer cells via induction of intracellular ROS accumulation (10), and the results of the present study were consistent with these results.

Apoptosis is a major process of programmed cell death, which involves two distinct apoptosis pathways including mitochondrial and death receptor pathways $(14,15)$. Abnormal ROS generation is a critical event during mitochondria-associated apoptosis. As vital signaling molecules, ROS regulate multiple intracellular signal transduction pathways that are involved in various cellular processes including cell proliferation, cell cycle progression, invasion, migration and apoptosis in cancer $(16,17)$. Our previous studies have demonstrated that JS-K, an important glutathione transferase-activated NO donor prodrug, was able to stimulate intercellular accumulation of ROS which resulted in cytotoxicity and apoptosis in cancer cells derived from human urogenital tumors $(10,18)$. In the present study, it was demonstrated that JS-K markedly increased intracellular ROS generation in Taxol-treated prostate cancer cells. It was also revealed that administration of the prooxidant GSSG exacerbated the inducing effect of JS-K on Taxol-induced apoptosis. However, the inducing effect of JS-K was inhibited in the presence of the antioxidant NAC. These data suggested that upregulated ROS generation may be a mechanism by which JS-K increases the anticancer activity of Taxol in prostate cancer cells.

GSH, as well as its oxidized form GSSG, are crucial elements involved in mitochondrial dysfunction, redox balance and apoptosis $(19,20)$. The physiological balance of GSH and GSSG is used to indicate the general cellular oxidation/reduction microenvironments and the redox balance (21). The results of the present study have demonstrated that JS-K significantly decreased the GSH/GSSG ratio, which resulted in a redox imbalance. Apoptosis induced by mitochondrial dysfunction is a fundamental apoptotic pathway accompanied by a series of events including decreases in mitochondrial membrane potential and ATP production, and activation of caspase-9 $(15,22,23)$. As an energy source in cellular metabolism, ATP production would be influenced by a decline in mitochondrial membrane potential during cellular apoptosis (24). The results of the present study demonstrated that JS-K significantly promoted Taxol-induced mitochondria-associated apoptosis, and exacerbated the decrease in mitochondrial membrane potential and ATP levels, and exacerbated the activation of caspase-9. In addition, Bcl-2 family members are important regulators of apoptosis, and dysregulation of Bcl-2 family members occurs in a number of diseases and stress-induced apoptosis $(25,26)$. As members of the Bcl-2 family, Bak, Bax and Bcl-2 serves crucial functions in mitochondrial stress-induced cellular apoptosis, and the appropriate balance of Bcl-2 and Bax is vital for cellular survival (27). In the present study, JS-K significantly exacerbated the Taxol-induced increase in expression levels of Bax and Bak proteins, and the decrease in expression of Bcl-2 protein. These results suggested that JS-K promoted Taxol-induced apoptosis through a mitochondria-mediated pathway.

In conclusion, the results of the present study indicated that the addition of JS-K increased the anticancer effect of Taxol on prostate cancer cells. It was revealed that the effects of combined treatment with JS-K and Taxol were reversed by an antioxidant and exacerbated by a pro-oxidant. It was hypothesized that ROS activation, induced by the combination of JS-K and Taxol, induced apoptosis in prostate cancer cells (Fig. 5). Further investigation is required to identify the roles and mechanisms underlying the combination of JS-K and Taxol.

\section{Acknowledgements}

Not applicable.

\section{Funding}

The present study was supported by The National Natural Science Funds (grant no. 81272833) of China.

\section{Availability of data and materials}

All data generated or analyzed during this study are included in this published article.

\section{Authors' contributions}

MNQ and JJL designed the experiments. MNQ, SZ, LZK, $\mathrm{HCT}$ and $\mathrm{XZ}$ performed the experiments. MNQ and JJL were involved in data and statistical analyses. MNQ and JJL wrote the article and prepared figures. JJL provided guidance and the financial support. All authors reviewed the manuscript.

\section{Ethics approval and consent to participate}

Not applicable.

\section{Patient consent for publication}

Not applicable.

\section{Competing interests}

The authors declare that they have no competing interests.

\section{References}

1. Weaver BA: How Taxol/paclitaxel kills cancer cells. Mol Biol Cell 25: 2677-2681, 2014.

2. Rowinsky EK, Cazenave LA and Donehower RC: Taxol: A novel investigational antimicrotubule agent. J Natl Cancer Inst 82: 1247-1259, 1990

3. Danesi R, Figg WD, Reed E and Myers CE: Paclitaxel (taxol) inhibits protein isoprenylation and induces apoptosis in PC-3 human prostate cancer cells. Mol Pharmacol 47: 1106-1111, 1995.

4. Obasaju $\mathrm{C}$ and Hudes GR: Paclitaxel and docetaxel in prostate cancer. Hematol Oncol Clin North Am 15: 525-545, 2001.

5. Kopczyńska E: Role of microRNAs in the resistance of prostate cancer to docetaxel and paclitaxel. Contemp Oncol (Pozn) 19: 423-427, 2015.

6. Sobue S, Mizutani N, Aoyama Y, Kawamoto Y, Suzuki M, Nozawa Y, Ichihara M and Murate T: Mechanism of paclitaxel resistance in a human prostate cancer cell line, PC3-PR, and its sensitization by cabazitaxel. Biochem Biophys Res Commun 479: 808-813, 2016.

7. Grimm EA, Sikora AG and Ekmekcioglu S: Molecular pathways: Inflammation-associated nitric-oxide production as a cancer-supporting redox mechanism and a potential therapeutic target. Clin Cancer Res 19: 5557-5563, 2013. 
8. Laschak M,Spindler KD, Schrader AJ,Hessenauer A, StreicherW, Schrader M and Cronauer MV: JS-K, a glutathione/glutathione S-transferase-activated nitric oxide releasing prodrug inhibits androgen receptor and WNT-signaling in prostate cancer cells. BMC Cancer 12: 130, 2012.

9. Tan G, Qiu M, Chen L, Zhang S, Ke L and Liu J: JS-K, a nitric oxide pro-drug, regulates growth and apoptosis through the ubiquitin-proteasome pathway in prostate cancer cells. BMC Cancer 17: 376, 2017.

10. Qiu M, Chen L, Tan G, Ke L, Zhang S, Chen H and Liu J: JS-K promotes apoptosis by inducing ROS production in human prostate cancer cells. Oncol Lett 13: 1137-1142, 2017.

11. Qiu M, Ke L, Zhang S, Zeng X, Fang Z and Liu J: JS-K, a GST-activated nitric oxide donor prodrug, enhances chemo-sensitivity in renal carcinoma cells and prevents cardiac myocytes toxicity induced by Doxorubicin. Cancer Chemother Pharmacol 80: 275-286, 2017.

12. Livak KJ and Schmittgen TD: Analysis of relative gene expression data using real-time quantitative PCR and the 2(-Delta Delta C(T)) method. Methods 25: 402-408, 2001

13. Wang SQ, Wang C, Chang LM, Zhou KR, Wang JW, Ke Y, Yang DX, Shi HG, Wang R, Shi XL, et al: Geridonin and paclitaxel act synergistically to inhibit the proliferation of gastric cancer cells through ROS-mediated regulation of the PTEN/PI3K/Akt pathway. Oncotarget 7: 72990-73002, 2016.

14. Ouyang L, Shi Z, Zhao S, Wang FT, Zhou TT, Liu B and Bao JK: Programmed cell death pathways in cancer: A review of apoptosis, autophagy and programmed necrosis. Cell Prolif 45: 487-498, 2012.

15. Elmore S: Apoptosis: A review of programmed cell death. Toxicol Pathol 35: 495-516, 2007.

16. Wu WS: The signaling mechanism of ROS in tumor progression. Cancer Metastasis Rev 25: 695-705, 2006.

17. Ray PD, Huang BW and Tsuji Y: Reactive oxygen species (ROS) homeostasis and redox regulation in cellular signaling. Cel Signal 24: 981-990, 2012.

18. Qiu M, Chen L, Tan G, Ke L, Zhang S, Chen H and Liu J: A reactive oxygen species activation mechanism contributes to JS-K-induced apoptosis in human bladder cancer cells. Sci Rep 5: 15104, 2015.
19. Nie F, Zhang X, Qi Q, Yang L, Yang Y, Liu W, Lu N, Wu Z, You Q and Guo Q: Reactive oxygen species accumulation contributes to gambogic acid-induced apoptosis in human hepatoma SMMC-7721 cells. Toxicology 260: 60-67, 2009.

20. Song X, Xie L, Wang X, Zeng Q, Chen TC, Wang W and Song X: Temozolomide-perillyl alcohol conjugate induced reactive oxygen species accumulation contributes to its cytotoxicity against non-small cell lung cancer. Sci Rep 6: 22762, 2016.

21. Brigelius-Flohé R and Maiorino M: Glutathione peroxidases. Biochim Biophys Acta 1830: 3289-3303, 2013.

22. Giampazolias E and Tait SW: Mitochondria and the hallmarks of cancer. FEBS J 283: 803-814, 2016.

23. Allan LA and Clarke PR: Apoptosis and autophagy: Regulation of caspase-9 by phosphorylation. FEBS J 276: 6063-6073, 2009.

24. Cosentino K and García-Sáez AJ: Mitochondrial alterations in apoptosis. Chem Phys Lipids 181: 62-75, 2014.

25. Kvansakul M and Hinds MG: The Bcl-2 family: Structures, interactions and targets for drug discovery. Apoptosis 20: 136-150, 2015.

26. Siddiqui WA, Ahad A and Ahsan H: The mystery of BCL2 family: Bcl-2 proteins and apoptosis: An update. Arch Toxicol 89: 289-317, 2015.

27. Tasyriq M, Najmuldeen IA, In LL, Mohamad K, Awang K and Hasima N: $7 \alpha$-hydroxy- $\beta$-sitosterol from chisocheton tomentosus induces apoptosis via dysregulation of cellular $\mathrm{Bax} / \mathrm{Bcl}-2$ ratio and cell cycle arrest by downregulating ERK1/2 activation. Evid Based Complement Alternat Med 2012: 765316 , 2012

This work is licensed under a Creative Commons Attribution-NonCommercial-NoDerivatives 4.0 International (CC BY-NC-ND 4.0) License. 